«Hormones et diabète »

\title{
Cibler la voie métabolique du cortisol comme action thérapeutique dans le diabète de type 2
}

\section{Targeting the metabolic pathway of cortisol as a potential therapy of type 2 diabetes}

\section{André J. Scheen}

Professeur ordinaire émérite,

Université de Liège :

Service de diabétologie, nutrition

et maladies métaboliques

et Unité de pharmacologie clinique

Département de médecine, CHU Liège, Belgique.
Sodetéé Journée thématique 2016 de la flabète Société francophone du diabète (SFD)

\section{Correspondance}

\section{André Scheen}

Département de Médecine

CHU Sart Tilman (B35)

4000 Liège - Belgique

andre.scheen@chu.ulg.ac.be

C 2016 - Elsevier Masson SAS - Tous droits réservés

\begin{abstract}
Résumé

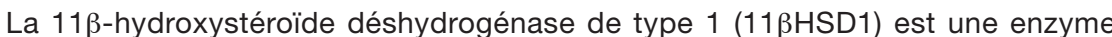
intervenant dans la transformation tissulaire de cortisone inactive en cortisol actif. Elle a été impliquée dans la physiopathologie de l'obésité abdominale, composante centrale du syndrome métabolique, une condition proche du syndrome de Cushing à certains égards. Des inhibiteurs synthétiques sélectifs de la 11ßHSD1 ont été testés dans des essais préliminaires chez des patients diabétiques de type 2 ou hypertendus, mais avec des résultats mitigés, jugés insuffisants en comparaison des résultats obtenus avec d'autres classes de médicaments déjà disponibles. Une étude récente dans la stéatose hépatique a donné des résultats prometteurs chez des sujets en surpoids ou obèses non diabétiques. Alors que certains inhibiteurs de la 11ßHSD1 ont été abandonnés, d'autres sont toujours en développement, ce qui témoigne de l'intérêt persistant pour cette approche thérapeutique innovante.
\end{abstract}

Mots-clés : Cortisol - diabète de type 2 - 11 $\beta$-hydroxystéroïde déshydrogénase de type 1 - obésité - stéatose hépatique.

\begin{abstract}
Summary
The 11ß-hydroxysteroid dehydrogenase type 1 (11ßHSD1) enzyme promotes the local conversion from inactive cortisone to active cortisol. It may play a role in the pathophysiology of abdominal obesity and the metabolic syndrome, both showing some similarities with the Cushing syndrome. Synthetic selective inhibitors of $11 \beta H S D 1$ have been investigated in pilot studies in overweight/obese patients with type 2 diabetes or hypertension. Although some positive effects were observed, they were considered as too modest compared to what could be obtained with other available drugs. More recently, a reduction in liver steatosis was reported in overweight/obese non-diabetic patients. Whereas some early $11 \beta H S D 1$ inhibitors have been abandoned, others are still in development, which demonstrates a persisting interest for this innovative therapeutic approach.
\end{abstract}

Key-words: Cortisol - 11 $\beta$-hydroxysteroid dehydrogenase type 1 - fatty liver disease - obesity - type 2 diabetes.

\section{Introduction}

- Le syndrome de Cushing, caractérisé par une sécrétion excessive de cortisol, combine plusieurs anomalies métaboliques dont une obésité centrale, une dysglycémie, et une hypertension artérielle (HTA). Ces anomalies se retrouvent dans le syndrome métabolique dont on connaît l'étroite relation avec l'obésité abdominale [1]. Le cortisol est une hormone de 
«Hormones et diabète»

contre-régulation, contrecarrant l'action de l'insuline. Par ailleurs, l'insulinorésistance est une anomalie importante, à la fois dans le syndrome métabolique et dans le diabète de type 2 (DT2). Bien que la cortisolémie et la cortisolurie soient, en général, normales ou à peine accrues dans l'obésité commune et le DT2, un hypercorticisme tissulaire local pourrait exister. En effet, l'enzyme $11 \beta$-hydroxystéroïde déshydrogénase de type 1 (11ßHSD1) est responsable de l'activation de cortisone en cortisol, en particulier dans le tissu adipeux et le foie. Un dysfonctionnement de la $11 \beta$ HSD1 pourrait résulter en une exposition de ces tissus à une concentration anormalement élevée de cortisol, ce qui pourrait entraîner diverses perturbations métaboliques. La $11 \beta$ HSD1 pourrait donc représenter une nouvelle cible thérapeutique chez le patient obèse avec un DT2 et/ou des troubles métaboliques associés [2-5].

- Nous avons déjà eu l'occasion de présenter, dans un article précédent paru en 2009 dans la revue Médecine des maladies Métaboliques, le rôle de la $11 \beta \mathrm{HSD} 1$ et de l'exposition tissulaire au cortisol dans le risque métabolique lié à l'obésité [6]. De même, nous avions à ce moment, dans un second article, présenté les premiers résultats d'une inhibition sélective de la $11 \beta$ HSD1 par des agents pharmacologiques en développement par diverses firmes pharmaceutiques pour traiter les anomalies métaboliques associées à l'obésité, en particulier le DT2 [7].

- Le présent article a pour but de brièvement résumer les données connues à l'époque et, surtout, de décrire les avancées dans ce domaine depuis 2009, avec, d'une part, les espoirs déçus dans le traitement du DT2 et, d'autre part, certaines nouvelles perspectives envisagées plus récemment pour les inhibiteurs de la $11 \beta$ HSD1.

\section{Distribution et rôle de la 11ßHSD1}

- L'enzyme 11ßHSD1 intervient dans la transformation de cortisone (inactive) en cortisol (actif) (figure 1) $[2,3,8]$. L'exposition tissulaire aux glucocorticoïdes est ainsi modulée localement, indépendamment des variations plasmatiques nycthémérales du cortisol. La distribution de la $11 \beta \mathrm{HSD} 1$ est ubiquitaire, puisque cette enzyme est présente dans le tissu adipeux, le foie, le muscle, le cerveau, le myocarde et la peau, notamment (figure 2). Dans le tissu adipeux, la 11ßHSD1 contrôlerait certaines régulations métaboliques à un niveau pré-récepteur et jouerait, en outre, un rôle capital dans la différenciation cellulaire [2, 3, 9]. Dans le foie, elle intervient dans le contrôle de la production hépatique du glucose, et son dysfonctionnement contribuerait à

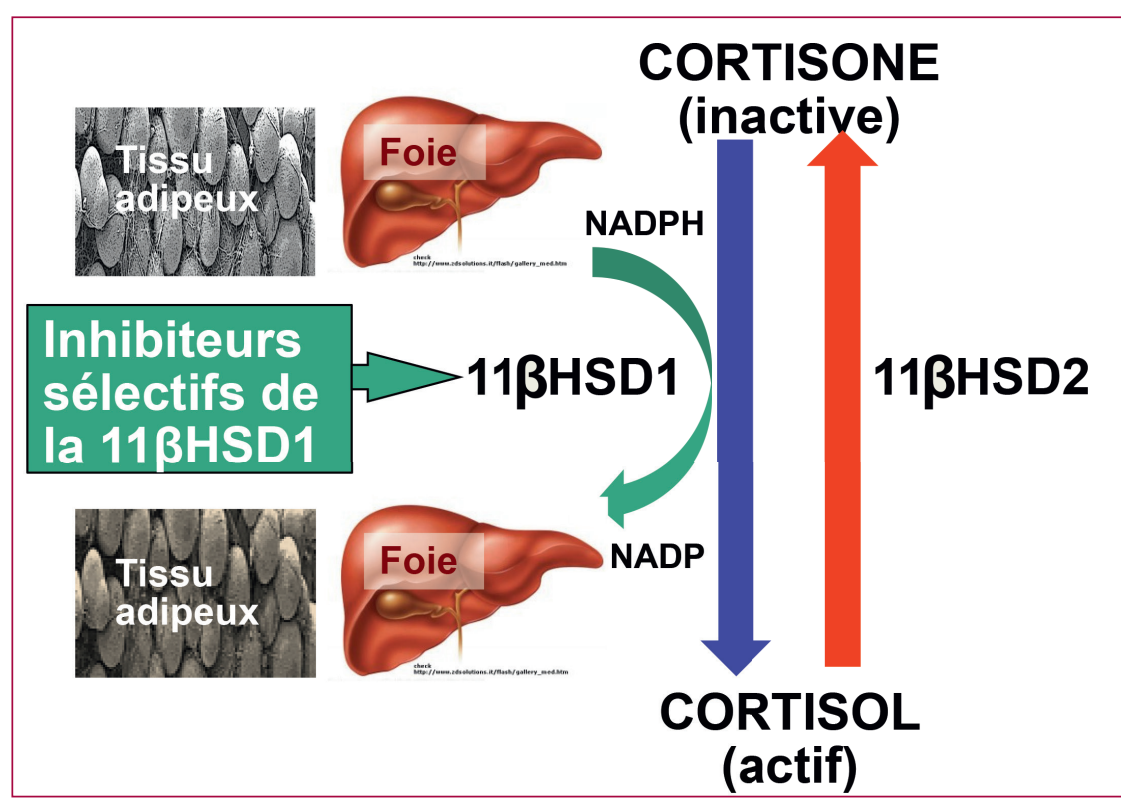

Figure 1. L'enzyme 11 $\beta$-hydroxystéroïde déshydrogénase de type 1 (11ßHSD1) transforme la cortisone, inactive sur le plan métabolique, en cortisol, métaboliquement actif, avec le NADPH comme cofacteur. À noter que l'enzyme 11 $\beta$-hydroxystéroïde déshydrogénase de type 2 (11ßHSD2) exerce une activité en sens opposé, notamment dans le rein.

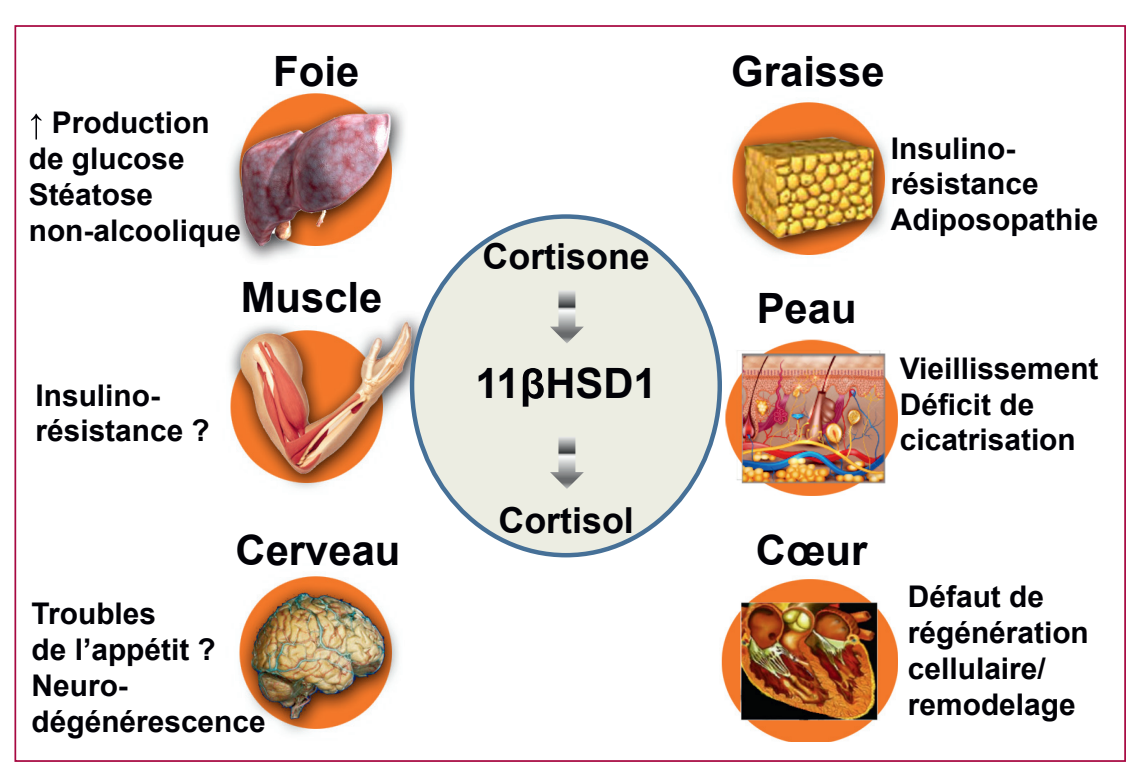

Figure 2. Schéma illustrant la distribution ubiquitaire de l'enzyme 11 $\beta$-hydroxystéroïde déshydrogénase de type 1 (11ßHSD1) et résumant quelques fonctions potentielles dans différents organes clé. 
l'apparition d'une stéatose hépatique [10]. Cette enzyme est également présente dans le cerveau, en particulier dans l'hypothalamus, où elle pourrait jouer un rôle dans les régulations métaboliques, dans le contrôle de l'appétit, et dans les rythmes circadiens [11]. La $11 \beta$ HSD1 pourrait être impliquée dans certaines maladies neurodégénératives ou psychiatriques [12]. La présence de la $11 \beta$ HSD1 a également été démontrée dans le myocarde où elle pourrait jouer un rôle dans les phénomènes de régénération cellulaire et de remodelage du cœur après un infarctus myocardique [13]. Enfin, la 11 $\beta$ HSD1 est également présente dans la peau, et une augmentation de son activité serait associée à un vieillissement de la peau et des problèmes de retard de cicatrisation, conditions également rencontrées chez les patients diabétiques [14].

- La régulation de cette enzyme se fait essentiellement par un contrôle de son expression, bien qu'une régulation post-transcriptionnelle ait également été proposée, via l'hexose-6-phosphate déshydrogénase (H6PDH) et l'approvisionnement en cofacteur (NADPH) (figure 1) [2, 3, 8]. La régulation de la $11 \beta$ HSD1 dépend de nombreux facteurs nutritionnels et hormonaux [2, 3, 9]. Elle peut être aussi altérée par des perturbateurs endocriniens de l'environnement, comme suggéré récemment [15].

- Certains effets hormonaux dépendent d'une modification de l'activité de $11 \beta$ HSD1. Ainsi, à titre d'exemple, il a été montré que l'hormone incrétine GIP (pour glucose-dependent insulinotropic polypeptide) inhibe la libération des acides gras par le tissu adipeux en réduisant l'expression et l'activité de la $11 \beta \mathrm{HSD} 1$ adipocytaire [16].

\section{Dysfonctionnement de la 11 $\beta$ HSD1 dans l'obésité}

- De nombreuses données animales sont en faveur d'une dysrégulation de la $11 \beta$ HSD1 en présence d'une obésité, et des modèles de knock-out (KO) ou de surexpression de cette enzyme ont confirmé son important rôle métabolique [2, 3, 8]. En 1997, une expression de la
$11 \beta$ HSD1 supérieure dans le tissu adipeux péri-viscéral a été retrouvée par rapport au tissu adipeux sous-cutané chez l'homme, ce qui a conduit au concept de «Cushing omental » [17]. Cependant, les études in vitro ultérieures ne se sont pas révélées univoques [2, 3, 8]. De même, les résultats des études in vivo, analysant les contributions respectives du tissu adipeux (viscéral ou sous-cutané) et du foie, chez l'homme de poids normal ou le sujet obèse, ont également donné des résultats assez disparates [18]. Une étude récente (BARICORT) a analysé les effets d'une chirurgie bariatrique chez le patient obèse [19]. L'importante perte de poids s'accompagne d'une réduction de l'activité de la 11ßHSD1 dans le tissu adipeux sous-cutané, mais d'une augmentation de l'activité de la $11 \beta \mathrm{HSD} 1$ dans le foie. À vrai dire, la cible tissulaire préférentielle (foie ou tissu adipeux) des inhibiteurs pharmacologiques de la $11 \beta H S D 1$ susceptibles d'être utilisés en thérapeutique chez l'homme reste discutée [2, 3]. Les données concernant le muscle sont moins nombreuses et non univoques. Une augmentation de l'expression de la $11 \beta \mathrm{HSD} 1$ a été rapportée dans les myotubes de patients DT2 [20], mais elle n'a pas été montrée altérée dans le muscle squelettique chez des sujets avec une obésité centrale et une insulinorésistance [21]. Enfin, le cerveau pourrait également jouer un rôle dans les dysrégulations métaboliques en relation avec la 11ßHSD1. Ainsi, une étude récente a suggéré une régulation négative de la $11 \beta$ HSD1 dans le cerveau de femmes en surpoids avec un DT2 [22]. - Une diminution d'activité de la $11 \beta$ HSD1 a été observée chez le sujet obèse non diabétique, mais pas chez le patient obèse DT2, où elle peut même être augmentée, en particulier dans le foie [23]. II a été suggéré qu'une réduction d'activité de la $11 \beta$ HSD1 pourrait être un mécanisme protecteur empêchant le sujet obèse d'évoluer vers un DT2. L'échec de ce processus pourrait mener progressivement au DT2 et aux anomalies métaboliques associées. II n'est donc pas étonnant qu'une modulation pharmacologique de la $11 \beta \mathrm{HSD} 1$ ait suscité l'intérêt de l'industrie pharmaceutique.

\section{Premiers essais d'inhibition pharmacologique de la 11ßHSD1 dans le diabète de type 2}

- Différents médicaments, utilisés dans le traitement des maladies métaboliques (fibrates, glitazones, metformine), de même que le salicylate, semblent diminuer l'activité de la $11 \beta H S D 1$, quoique de façon variable [2, 3]. Les premiers essais chez l'homme ont fait appel à la carbénoxolone, mais les résultats ont été peu encourageants dans plusieurs essais pilotes chez des sujets avec une certaine insulinorésistance et la présence ou non d'un DT2 [24]. Les raisons invoquées pour cet échec relatif étaient que la carbénoxolone n'est pas un inhibiteur puissant de la $11 \beta \mathrm{HSD} 1$ et que, par ailleurs, elle n'exerce pas une action suffisamment sélective, puisque qu'elle inhibe également l'enzyme 11 $\beta$-hydroxystéroïde déshydrogénase de type 2 (11ßHSD2) [24] (figure 1). L'industrie pharmaceutique a tenté de développer des inhibiteurs synthétiques, plus puissants et plus sélectifs, de la $11 \beta$ HSD1 $[25,26]$, dont quelques-uns ont été testés chez I'homme, en particulier le INCB013739 (Incyte Corporation, USA), le MK-0916 (Merck Sharp \& Dohme Corp., USA), et le MK-0736 (Merck Sharp \& Dohme Corp., USA).

- Une étude pilote (uniquement publiée sous la forme d'un résumé de congrès) a montré une amélioration de la sensibilité hépatique et périphérique à l'insuline, mesurée avec la technique de référence du "glucose clamp ", ainsi qu'une diminution de la glycémie à jeun (non statistiquement significative, cependant), du cholestérol LDL et de la pression artérielle chez des sujets obèses DT2 traités pendant 28 jours par le INCB013739 (tableau I) [27].

- Dans une étude contrôlée de 12 semaines versus placebo, chez des patients DT2 traités par metformine, une réduction significative du taux d'hémoglobine glyquée $\left(\mathrm{HbA}_{1 \mathrm{c}}\right)$, de l'ordre de 0,4-0,5\% avec une dose orale de 100200 mg par jour d'INCB013739, a été observée. Par ailleurs, le traitement par inhibiteur de la 11ßHSD1 a été associé 
« Hormones et diabète »

à une légère diminution pondérale, une baisse de la glycémie à jeun, une réduction des marqueurs d'insulinorésistance, et une certaine amélioration du profil lipidique (tableau I) [28].

- Un autre inhibiteur de la 11ßHSD1 a été testé chez l'homme, le MK-0916 [29]. Des résultats encourageants ont été observés chez le patient obèse DT2 traité par le MK-0916, avec une réduction de $-0,3 \%$ du taux d'HbA1c, associée à une diminution du poids et de la pression artérielle [30]. Les effets positifs d'un traitement de 12 semaines par le MK-0916 ont été confirmés chez le patient obèse hypertendu sans DT2 en ce qui concerne la perte de poids et la diminution de la pression artérielle [31]. - Dans la même étude, un autre inhibiteur de la 11ßHSD1, le MK-0736, a également donné des résultats favorables, toujours chez le patient obèse hypertendu non diabétique, avec la mise en évidence d'une légère réduction du poids corporel, de la pression artérielle, et du taux de cholestérol LDL (tableau II) [31].

- Ces essais démontrent l'intérêt thérapeutique potentiel des inhibiteurs sélectifs de la $11 \beta$ HSD1 dans la prise en charge de l'obésité compliquée d'un DT2 ou d'une HTA et, d'une façon plus large, dans le traitement du syndrome métabolique [32, 33]. Cependant, globalement, les résultats cliniques ont été jugés insuffisants par comparaison à ceux obtenus avec d'autres classes de médicaments déjà disponibles, en particulier face à l'émergence des nouveaux antidiabétiques oraux représentés par les inhibiteurs de la dipeptidyl peptidase-4 (gliptines) et les inhibiteurs des co-transporteurs sodium-glucose de type 2 (gliflozines) [34].

- Comment expliquer ces résultats finalement assez décevants chez l'homme, alors que les premiers essais chez le rongeur, en particulier la souris, s'étaient révélés particulièrement prometteurs [24] ? Cet échec relatif pourrait résulter d'un manque de sélectivité des inhibiteurs de la 11ßHSD1 utilisés jusqu'à présent, qui exercent aussi une action sur la 11 $\beta$-réductase [35]. De plus, la régulation de l'activité de la $11 \beta \mathrm{HSD} 1$ est particulièrement complexe et est conditionnée par la disponibilité en NADPH tissulaire, de telle sorte qu'il

Tableau I. Résumé des effets métaboliques obtenus avec l'inhibiteur sélectif de l'enzyme

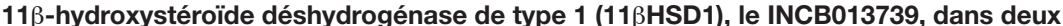
essais cliniques réalisés chez le patient diabétique de type 2.

Les résultats sont exprimés par les différences par rapport au groupe placebo (seules les valeurs de $p<0,10$ sont indiquées).

\begin{tabular}{|lccc|} 
& $\begin{array}{c}\text { Étude pilote } \\
\text { Hawkins et al. 2008 } \\
{[27]}\end{array}$ & $\begin{array}{c}\text { Étude contrôlée versus placebo } \\
\text { Rosenstock et al. 2010 [28] }\end{array}$ \\
\hline $\mathrm{n}$ & 30 & 27 & 27 \\
\hline Durée (semaines) & 4 & 12 & 12 \\
\hline Dose INCB013739 (mg/jour) & $2 \times 100$ & $1 \times 100$ & $1 \times 200$ \\
\hline Poids corporel (kg) & $\mathrm{ND}$ & $-1,1(p<0,05)$ & $-0,9(p<0,05)$ \\
\hline Glycémie à jeun $(\mathrm{mg} / \mathrm{dL})$ & $-18,1$ & $-1,6$ & $-11,5(p<0,01)$ \\
\hline $\mathrm{HbA}_{1 \mathrm{t}}(\%)$ & $\mathrm{ND}$ & $-0,47(p<0,05)$ & $-0,56(p<0,01)$ \\
\hline Insulinosensibilité & Améliorée dans le foie & Améliorée & Améliorée \\
Cholestérol LDL $(\mathrm{mg} / \mathrm{dL})$ & (technique du clamp) & (méthode HOMA) & $+24 \%(p<0,05)$ \\
\hline Cholestérol HDL $(\mathrm{mg} / \mathrm{dL})$ & $-20,1(p=0,001)$ & $-4,6(p<0,10)$ & $-4,3$ \\
\hline Triglycérides $(\mathrm{mg} / \mathrm{dL})$ & $-2,0$ & $+0,4$ & $+0,8$ \\
\hline PA systolique $(\mathrm{mm} \mathrm{Hg})$ & $-12,8$ & $-11,5$ & $-10,6$ \\
\hline PA diastolique $(\mathrm{mm} \mathrm{Hg})$ & $-6,4(p=\mathrm{ND})$ & $+0,5$ & $-0,4$ \\
\hline
\end{tabular}

HOMA : homeostasis model assessment ; ND : non disponible.

Remarque : les diminutions observées dans l'étude contrôlée en ce qui concerne la glycémie à jeun, le taux d'HbA $\mathrm{A}_{1 c}$, le cholestérol LDL, et les concentrations de triglycérides, ont été plus importantes si les valeurs à l'inclusion étaient plus élevées, respectivement, > $160 \mathrm{mg} / \mathrm{dL}, \geq 8 \%$, $\geq 130 \mathrm{mg} / \mathrm{dL}$, et $\geq 200 \mathrm{mg} / \mathrm{dL}$.

Tableau II. Résumé des effets métaboliques obtenus avec deux inhibiteurs de l'enzyme 11 $\beta$-hydroxystéroïde déshydrogénase de type 1 (11ßHSD1), le MK-0916 et le MK-0736, dans deux essais cliniques de 12 semaines contrôlés versus placebo réalisés chez le patient obèse diabétique de type 2 et chez le patient obèse hypertendu.

Les résultats sont exprimés par les différences par rapport au groupe placebo (seules les valeurs de $p<0,10$ sont indiquées)

\begin{tabular}{|c|c|c|c|}
\hline & $\begin{array}{l}\text { Feig et al. } \\
2011 \text { [30] }\end{array}$ & $\begin{array}{l}\text { Shah et al. } \\
2011 \text { [31] }\end{array}$ & $\begin{array}{l}\text { Shah et al. } \\
2011 \text { [31] }\end{array}$ \\
\hline Patients & Obèses, DT2 & Obèses, hypertendus & Obèses, hypertendus \\
\hline $\mathrm{n}$ & $\begin{array}{c}\mathrm{n}=38 \text { vs } \mathrm{n}=37 \\
\text { sous placebo }\end{array}$ & $\begin{array}{c}\mathrm{n}=49 \text { vs } \mathrm{n}=51 \\
\text { sous placebo }\end{array}$ & $\begin{array}{c}\mathrm{n}=52 \text { vs } \mathrm{n}=51 \\
\text { sous placebo }\end{array}$ \\
\hline Molécule/dose & $\begin{array}{l}\text { MK-0916 } \\
6 \text { mg/jour }\end{array}$ & $\begin{array}{l}\text { MK-0916 } \\
6 \mathrm{mg} / \mathrm{jour}\end{array}$ & $\begin{array}{l}\text { MK-0736 } \\
7 \text { mg/jour }\end{array}$ \\
\hline Poids corporel (kg) & $-1,8(p<0,001)$ & $-1,7(p<0,001)$ & $-1,4(p<0,001)$ \\
\hline Tour de taille $(\mathrm{cm})$ & $-1,6(p=0,064)$ & $-1,4$ & $-1,8(p=0,059)$ \\
\hline Glycémie à jeun (mg/dL) & $-5,4$ & ND & ND \\
\hline $\begin{array}{l}\text { Glycémie post-prandiale } \\
\text { (mg/dL) }\end{array}$ & $-12,6$ & ND & ND \\
\hline $\mathrm{HbA}_{1 \mathrm{c}}(\%)$ & $-0,3(p=0,049)$ & ND & ND \\
\hline Cholestérol LDL (mg/dL) & $+10,4(p=0,041)$ & $-4,5$ & $-12,3(p=0,005)$ \\
\hline Cholestérol HDL (mg/dL) & $-3,0$ & $+2,1$ & $-6,3(p=0,015)$ \\
\hline Triglycérides (mg/dL) & $+11,5$ & $+2,3$ & $+4,9$ \\
\hline PA systolique (mm Hg) & $-7,9(p<0,001)$ & $-4,1(p=0,099)$ * & $-4,9(p=0,046)$ * \\
\hline PA diastolique $(\mathrm{mm} \mathrm{Hg})$ & $-5,4(p<0,001)$ & $-3,2(p=0,042)$ * & $-2,2^{*}$ \\
\hline
\end{tabular}

DT2 : diabète de type 2 ; ND : non disponible ; PA : pression artérielle.

* Diminutions de la PA systolo-diastolique confirmées par un monitoring ambulatoire de la PA de 24 heures dans cette étude.

peut exister, selon les circonstances, un recyclage entre cortisol et cortisone [35]. Une autre explication pourrait se trouver dans des différences inter-espèces. Ainsi, il a été démontré qu'une inhibition continue de la $11 \beta$ HSD1 dans le tissu adipeux entraîne une tachyphylaxie chez l'homme (et chez le rat), alors que semblable phénomène d'échappement n'est pas observé chez la souris [36]. 


\section{Essais récents dans le traitement de la stéatose hépatique}

- Alors que les essais dans le traitement du DT2 sont actuellement interrompus avec les inhibiteurs de la $11 \beta \mathrm{HSD} 1$, de nouvelles perspectives apparaissent, en particulier en ce qui concerne la prise en charge de la stéatose hépatique.

- Une étude récente, chez l'homme, des « single nucleotide polymorphisms " (SNPs) dans le gène codant pour la $11 \beta$ HSD1 a montré que cette enzyme pourrait jouer un rôle non négligeable dans la pathogénie de la stéatose non alcoolique associée à l'obésité viscérale, et constituer ainsi une cible thérapeutique potentielle dans cette pathologie [10].

- Chez la souris $d b / d b$, une étude a montré qu'un agent inhibant la $11 \beta \mathrm{HSD} 1$ protège contre l'insulinorésistance et la stéatose hépatique [37]. Ces résultats encourageants ont conduit à la réalisation d'un essai clinique contrôlé pilote chez l'homme [38]. Dans cette étude de phase $\mathrm{lb}$, des patients non diabétiques, avec un surpoids ou une obésité (indice de masse corporelle $[\mathrm{IMC}]>27 \mathrm{~kg} / \mathrm{m}^{2}$ ), avec une insulinorésistance appréciée par l'index Homeostasis model assessment (HOMA) et une stéatose hépatique quantifiée par spectroscopie en résonance magnétique, ont été traités, de façon aléatoire et en double insu, soit par un inhibiteur de la 11 $\beta$ HSD1 (RO5093151 per os, 200 mg deux fois par jour ; F. Hoffmann-La Roche, Bâle, Suisse) $(n=41)$, soit par un placebo $(n=41)$, pendant une période de 12 semaines. Le contenu moyen en graisse du foie a diminué significativement dans le groupe R05093151 (de 16,75\% à $14,28 \%)$, mais pas dans le groupe placebo (de 18,53\% à 18,46\%; $p$ $=0,02$ pour la différence entre les deux groupes). Ces résultats, certes encore préliminaires et obtenus sur une durée de traitement limitée, suggèrent que le R05093151 est capable de réduire le contenu graisseux hépatique [38].

- L'approche d'inhibition pharmacologique de la $11 \beta \mathrm{HSD} 1$ mérite d'être évaluée plus avant dans un essai de plus longue durée dans cette indication spécifique de la stéatose non alcoolique, une pathologie fréquemment observée chez les patients avec obésité viscérale et/ou un DT2 [39].

\section{Autres perspectives dans les maladies dégénératives liées à l'âge}

- Le vieillissement s'accompagne de diverses complications, dont la dysglycémie (voire le DT2) et l'HTA, deux composantes du syndrome métabolique, et l'athérosclérose avec ses complications cardiovasculaires. Par ailleurs, il existe certaines similitudes entre les complications liées au diabète et celles liées à l'âge avec, outre les maladies vasculaires, une augmentation des pathologies neurodégénératives [40]. Le cerveau joue un rôle central dans le contrôle des régulations métaboliques [40], et la présence de la $11 \beta$ HSD1 dans l'hypothalamus suggère que cette enzyme pourrait jouer un rôle dans le contrôle de l'appétit [11]. La présence de la $11 \beta$ HSD1 dans le cerveau est connue depuis au moins une vingtaine d'années [12], et son rôle dans certaines anomalies du fonctionnement cérébral est suspectée [41].

- La $11 \beta$ HSD1 est donc susceptible d'intéresser également les neurologues et les psychiatres. Dans ce domaine, les études chez l'homme ont principalement concerné un autre inhibiteur de la $11 \beta$ HSD1 que ceux précédemment mentionnés dans les études métaboliques, à savoir l'ABT-384 (Abbott, USA) [42, 43]. Cependant, une étude récente, réalisée chez des patients avec une maladie d'Alzheimer, n'a pas montré d'amélioration avec un traitement par ABT-384 pendant 12 semaines par rapport à un placebo alors que, parallèlement, un traitement par donépézil entraînait une certaine amélioration du score de performance cognitive [44].

\section{Nouveaux inhibiteurs de la 11 $\beta$ HSD1 en développement}

De nouveaux inhibiteurs de la $11 \beta$ HSD1 sont toujours en développement, pour la plupart à une phase encore relativement précoce $[3,26,32,45]$. Ceci démontre qu'il persiste un intérêt pour cette approche thérapeutique qui consiste à inhiber l'activité de cette enzyme. Néanmoins, le chemin sera sans doute encore long avant qu'un inhibiteur spécifique de la 11ßHSD1 puisse être utilisé un jour en clinique, en particulier dans le domaine des maladies métaboliques où

\section{Les points essentiels}

- L'obésité abdominale est commune aux patients diabétiques de type 2 (DT2) et aux patients avec un syndrome de Cushing, qui partagent, par ailleurs, certaines anomalies caractéristiques du syndrome métabolique.

- Un hypercorticisme tissulaire chez le sujet obèse a été suspecté avec la mise en évidence d'une enzyme appelée $11 \beta$-hydroxystéroïde déshydrogénase de type 1 (11ßHSD1) qui transforme la cortisone inactive en cortisol actif.

- Un dysfonctionnement de cette 11ßHSD1 a été mis en évidence dans divers modèles animaux caractérisés par une obésité et une insulinorésistance, et dans le tissu adipeux et le foie de patients avec obésité abdominale, syndrome métabolique et DT2. - Quelques essais cliniques avec des inhibiteurs relativement sélectifs de la 11/BHSD1 ont montré, chez des patients obèses avec un DT2 ou une hypertension artérielle, des effets favorables sur le poids corporel, les anomalies glycémiques, la cholestérolémie et la pression artérielle, mais d'amplitude relativement modeste.

- Alors que les essais avec les premiers inhibiteurs de la 11ßHSD1 ont été abandonnés dans le traitement du DT2, un essai récent s'est révélé prometteur dans le traitement de la stéatose hépatique non alcoolique.

- De nouveaux inhibiteurs de la 11ßHSD1 sont toujours en cours de développement, et des avancées sont encore attendues dans ce domaine dans les prochaines années. 
«Hormones et diabète »

la diversité des approches pharmacologiques s'est considérablement amplifiée ces dernières années [34].

\section{NOTE}

Ce texte correspond à la présentation de l'auteur lors de la Journée Thématique 2016 de la Société francophone du diabète (SFD) « Hormones et diabète ", Paris, 9 décembre 2016.

\section{Déclaration d'intérêt}

L'auteur ne déclare pas de conflit d'intérêt en lien direct avec cet article. II déclare avoir reçu des honoraires, à titre personnel ou institutionnel, comme orateur, conseiller scientifique et/ou investigateur clinicien, de la part des firmes suivantes : AstraZeneca, Boehringer Ingelheim, Eli Lilly, GlaxoSmithKline, Janssen, Merck Sharp \& Dohme (MSD), Novartis, Novo Nordisk et Sanofi.

\section{Références}

[1] Anagnostis P, Athyros VG, Tziomalos K, et al. Clinical review: The pathogenetic role of cortisol in the metabolic syndrome: a hypothesis. J Clin Endocrinol Metab 2009;94:2692-701.

[2] Tomlinson JW, Walker EA, Bujalska IJ, et al. $11 \beta$-hydroxysteroid dehydrogenase type 1 : a tissue-specific regulator of glucocorticoid response. Endocr Rev 2004;25:831-66.

[3] Gathercole LL, Lavery GG, Morgan SA, et al. $11 \beta$-Hydroxysteroid dehydrogenase 1 : translational and therapeutic aspects. Endocr Rev 2013;34:525-55.

[4] Morton NM. Obesity and corticosteroids: $11 \beta$-hydroxysteroid type 1 as a cause and therapeutic target in metabolic disease. Mol Cell Endocrinol 2010;316:154-64.

[5] Hollis G, Huber R. 11 1 -Hydroxysteroid dehydrogenase type 1 inhibition in type 2 diabetes mellitus. Diabetes Obes Metab 2011;13:1-6.

[6] lovino A, Scheen AJ. La 11 $\beta$-hydroxystéroide déshydrogénase de type 1 - $1^{\text {re }}$ partie : rôle de l'exposition tissulaire au cortisol dans le risque métabolique lié à l'obésité. Médecine des maladadies Métaboliques 2009;3:507-13.

[7] lovino A, Scheen AJ. La 11 $\beta$-hydroxystéroïde déshydrogénase de type $1-2^{e}$ partie : Inhibition sélective pour traiter les anomalies métaboliques associées à l'obésité. Médecine des maladies Métaboliques 2009;3:595-600.

[8] Woods C, Tomlinson JW. The dehydrogenase hypothesis. Adv Exp Med Biol 2015;872:353-80.

[9] Stulnig TM, WaldhäusIW. 11ß-Hydroxysteroid dehydrogenase Type 1 in obesity and Type 2 diabetes. Diabetologia 2004;47:1-11.

[10] Lutz SZ, Peter A, Machicao F, et al. Genetic variation in the $11 \beta$-hydroxysteroiddehydrogenase 1 gene determines NAFLD and visceral obesity. J Clin Endocrinol Metab 2016 Oct 7 [Epub ahead of print].

[11] Bisschop PH, Dekker MJ, Osterthun W, et al. Expression of $11 \beta$-hydroxysteroid

\section{Conclusion}

L'étonnante similitude existant entre le syndrome de Cushing et le syndrome métabolique associé à l'obésité abdominale a suscité l'intérêt pour la 11ßHSD1, une enzyme qui transforme la cortisone inactive en cortisol métaboliquement actif au niveau tissulaire. Les études réalisées, même si elles sont assez disparates, plaident pour une dysfonction de la $11 \beta H S D 1$, en particulier dans le tissu adipeux et le foie, chez les sujets obèses et/ ou DT2. Les premiers essais réalisés avec de petites molécules inhibant la 11ßHSD1 ont, certes, prouvé le concept de cette approche pharmacologique innovante. Cependant, les résultats obtenus sur le plan des améliorations métaboliques n'ont pas été d'une amplitude jugée suffisante pour poursuivre les investigations dans le DT2. Néanmoins, d'autres perspectives peuvent être envisagées, parmi lesquelles le traitement de la stéatose hépatique non alcoolique, fréquemment observée chez les patients avec une obésité viscérale ou un DT2 mal équilibré. Le rôle de la 11ßHSD1 dans certaines maladies neurodégénératives représente également une ouverture pour de nouvelles indications thérapeutiques pour les inhibiteurs de la 11ßHSD1 en développement.

dehydrogenase type 1 in the human hypothalamus. J Neuroendocrinol 2013;25:425-32.

[12] Seckl JR. 11ß-Hydroxysteroid dehydrogenase in the brain: a novel regulator of glucocorticoid action? Front Neuroendocrinol 1997;18:49-99.

[13] Gray GA, White Cl, Castellan RF, et al. Getting to the heart of glucocorticoid regeneration: $11 \beta-H S D 1$ and the myocardium. J Mol Endocrinol 2016 Aug 23 [Epub ahead of print].

[14] Terao M, Katayama I. Local cortisol/corticosterone activation in skin physiology and pathology. J Dermatol Sci 2016;84:11-6.

[15] Vitku J, Starka L, Bicikova M, et al. Endocrine disruptors and other inhibitors of $11 \beta$-hydroxysteroid dehydrogenase 1 and 2: Tissue-specific consequences of enzyme inhibition. J Steroid Biochem Mol Biol 2016;155:207-16.

[16] Gögebakan Ö, Andres J, Biedasek K, et al. Glucose-dependent insulinotropic polypeptide reduces fat-specific expression and activity of $11 \beta$-hydroxysteroid dehydrogenase type 1 and inhibits release of free fatty acids. Diabetes 2012;61:292-300.

[17] Bujalska IJ, Kumar S, Stewart PM. Does central obesity reflect "Cushing's disease of the omentum»? Lancet 1997;349:1210-3.

[18] Valsamakis G, Anwar A, Tomlinson JW, et al. 11 $\beta$-hydroxysteroid dehydrogenase type 1 activity in lean and obese males with type 2 diabetes mellitus. J Clin Endocrinol Metab 2004;89:4755-61.

[19] Woods CP, Corrigan M, Gathercole L, et al. Tissue specific regulation of glucocorticoids in severe obesity and the response to significant weight loss following bariatric surgery (BARICORT). J Clin Endocrinol Metab 2015;100:1434-44.

[20] Abdallah BM, Beck-Nielsen H, Gaster M. Increased expression of $11 \beta$-hydroxysteroid dehydrogenase type 1 in type 2 diabetic myotubes. Eur J Clin Invest 2005;35:627-34.

[21] Inder WJ, Obeyesekere VR, Alford FP, Jang C. Skeletal muscle $11 \beta \mathrm{HSD} 1$ activity of nondiabetic subjects is unaltered in central obesity-associated insulin resistance. Horm Metab Res 2011;43:257-60.

[22] Li S, Liao Y, Wang L, et al. Dysregulation of hypothalamo-pituitary-adrenocortical axis in overweight female diabetic subjects is associated with downregulation of corticosteroid receptors and $11 \beta-H S D 1$ in the brain. Horm Metab Res 2016;48:178-84.

[23] Stimson RH, Andrew R, McAvoy NC, et al. Increased whole-body and sustained liver cortisol regeneration by $11 \beta$-hydroxysteroid dehydrogenase type 1 in obese men with type 2 diabetes provides a target for enzyme inhibition. Diabetes 2011;60:720-5.

[24] Stomby A, Andrew R, Walker BR, Olsson T. Tissue-specific dysregulation of cortisol regeneration by $11 \beta \mathrm{HSD} 1$ in obesity: has it promised too much? Diabetologia 2014;57:1100-10.

[25] Wang M. Inhibitors of $11 \beta$-hydroxysteroid dehydrogenase type 1 in antidiabetic therapy. Handb Exp Pharmacol 2011;(203):127-46.

[26] Anagnostis P, Katsiki N, Adamidou F, et al 11 beta-Hydroxysteroid dehydrogenase type 1 inhibitors: novel agents for the treatment of metabolic syndrome and obesity-related disorders? Metabolism 2013;62:21-33.

[27] Hawkins M, Hunter DJ, Kishore P, et al. INCB013739, a selective inhibitor of $11 \beta$-hydroxysteroid dehydrogenase type 1 (11ßHSD1), improves insulin sensitivity and lowers plasma cholesterol over 28 days in patients with type 2 diabetes mellitus (abstract). Diabetes 2008;57(Suppl.1):A99-A100 [Abstract].

[28] Rosenstock J, Banarer S, Fonseca VA, et al; INCB1379-202 Principal Investigators. The 11 - $\beta$-hydroxysteroid dehydrogenase type 1 inhibitor INCB13739 improves hyperglycemia in patients with type 2 diabetes inadequately controlled by metformin monotherapy. Diabetes Care 2010;33:1516-22.

[29] Wright DH, Stone JA, Crumley TM, et al. Pharmacokinetic-pharmacodynamic studies of the 11ß-hydroxysteroid dehydrogenase type 1 inhibitor MK-0916 in healthy subjects. Br J Clin Pharmacol 2013;76:917-31. 
[30] Feig PU, Shah S, Hermanowski-Vosatka A, et al. Effects of an $11 \beta$-hydroxysteroid dehydrogenase type 1 inhibitor, MK-0916, in patients with type 2 diabetes mellitus and metabolic syndrome. Diabetes Obes Metab 2011;13:498-504.

[31] Shah S, Hermanowski-Vosatka A, Gibson $\mathrm{K}$, et al. Efficacy and safety of the selective $11 \beta-H S D-1$ inhibitors MK-0736 and MK-0916 in overweight and obese patients with hypertension. J Am Soc Hypertens 2011;5:166-76.

[32] Anderson A, Walker BR. 11 $\beta$-HSD1 inhibitors for the treatment of type 2 diabetes and cardiovascular disease. Drugs 2013;73:1385-93.

[33] Schnackenberg CG, Costell MH, Krosky DJ, et al. Chronic inhibition of $11 \beta$-hydroxysteroid dehydrogenase type 1 activity decreases hypertension, insulin resistance, and hypertriglyceridemia in metabolic syndrome. Biomed Res Int 2013;2013:427640.

[34] Scheen AJ. Antidiabétiques oraux dans le traitement du diabète de type 2 : perspectives historique et médico-économique. Médecine des maladies Métaboliques 2015;9:186-97.

[35] Hughes KA, Manolopoulos KN, lqbal J, et al. Recycling between cortisol and cortisone in human splanchnic, subcutaneous adipose, and skeletal muscle tissues in vivo. Diabetes 2012;61:1357-64.

[36] Morentin Gutierrez P, Gyte A, deSchoolmeester $\mathrm{J}$, et al. Continuous inhibition of $11 \beta$-hydroxysteroid dehydrogenase type I in adipose tissue leads to tachyphylaxis in humans and rats but not in mice. Br J Pharmacol 2015;172:4806-16.

[37] Yuan X, Li H, Bai H, et al. The 11 $\beta$-hydroxysteroid dehydrogenase type 1 inhibitor protects against the insulin resistance and hepatic steatosis in $d b / d b$ mice. Eur J Pharmacol 2016;788:140-51.

[38] Stefan N, Ramsauer M, Jordan P, et al. Inhibition of $11 \beta$-HSD1 with R05093151 for non-alcoholic fatty liver disease: a multicentre, randomised, double-blind, placebo-controlled trial. Lancet Diabetes Endocrinol 2014;2:406-16.

[39] Luyckx FH, Lefebvre PJ, Scheen AJ. Nonalcoholic steatohepatitis: association with obesity and insulin resistance, and influence of weight loss. Diabetes Metab 2000;26:98-106.

[40] Scheen AJ. Central nervous system: a conductor orchestrating metabolic regulations harmed by both hyperglycaemia and hypoglycaemia. Diabetes Metab 2010;36(Suppl.3):S31-8.

[41] Martocchia A, Stefanelli M, Falaschi GM, et al. Recent advances in the role of cortisol and metabolic syndrome in age-related degenerative diseases. Aging Clin Exp Res 2016;28:17-23.

[42] Liu W, Katz DA, Locke C, et al. Clinical safety, pharmacokinetics, and pharmacodynamics of the $11 \beta$-hydroxysteroid dehydrogenase type 1 inhibitor ABT-384 in healthy volunteers and elderly adults. Clin Pharmacol Drug Dev 2013;2:133-51.

[43] Katz DA, Liu W, Locke C, et al. Periphera and central nervous system inhibition of $11 \beta$-hydroxysteroid dehydrogenase type 1 in man by the novel inhibitor ABT-384. Transl Psychiatry 2013;3:e295.

[44] Marek GJ, Katz DA, Meier A, et al. Efficacy and safety evaluation of HSD-1 inhibitor ABT-384 in Alzheimer's disease. Alzheimers Dementia 2014;10(5 Suppl):S364-73.

[45] Scott JS, Goldberg FW, Turnbull AV. Medicinal chemistry of inhibitors of $11 \beta$-hydroxysteroid dehydrogenase type 1 (11 $\beta$-HSD1). J Med Chem 2014;57:4466-86 